\title{
Spin wave excitations in the pyrovanadate $\alpha-\mathrm{Cu}_{2} \mathrm{~V}_{2} \mathrm{O}_{7}$
}

\author{
A. Banerjee ${ }^{1}$, J. Sannigrahi ${ }^{1}$, S. Bhowal ${ }^{1}$, I. Dasgupta ${ }^{1}$, S. Majumdar ${ }^{1}$, \\ H. C. Walker ${ }^{2}$, A. Bhattacharyya ${ }^{2,3,4}$, and D. T. Adroja ${ }^{2,3}$ \\ ${ }^{1}$ Department of Solid State Physics, Indian Association for the Cultivation of Science, \\ $2 A \&$ B Raja S. C. Mullick Road, Jadavpur, Kolkata 700 032, India \\ ${ }^{2}$ ISIS Facility, Rutherford Appleton Laboratory, Didcot, United Kingdom \\ ${ }^{3}$ Highly Correlated Matter Research Group, Physics Department, \\ University of Johannesburg, Auckland Park 2006, South Africa and \\ ${ }^{4}$ Department of Physics, Ramakrishna Mission Vivekananda University, \\ Belur Math, Howrah 711202, West Bengal, India
}

\begin{abstract}
The pyrovanadate $\alpha-\mathrm{Cu}_{2} \mathrm{~V}_{2} \mathrm{O}_{7}$ belongs to the orthorhombic ( $F d d 2$ ) class of crystals with noncentrosymmetric crystal structure. Recently, the compound has been identified to be a magnetic multiferroic with a substantial electric polarization below the magnetic transition temperature $T_{C}=$ $35 \mathrm{~K}$. Here we report the results of our inelastic neutron scattering (INS) studies on a polycrystalline sample of $\alpha-\mathrm{Cu}_{2} \mathrm{~V}_{2} \mathrm{O}_{7}$. Our INS data clearly show the existence of dispersive spin wave excitations below $T_{C}$ with a zone-boundary energy of $11 \mathrm{meV}$ at $5 \mathrm{~K}$. We have analyzed the data using linear spin wave theory, which shows good agreement between the experiment and calculation. The analysis is consistent with the third nearest neighbor exchange interaction playing a dominant role in the magnetism of the material.
\end{abstract}

PACS numbers: 78.70.Nx, 75.85.+t, 75.30.Ds

The family of pyrovanadates with general formula $\mathrm{M}_{2} \mathrm{~V}_{2} \mathrm{O}_{7}(\mathrm{M}=\mathrm{Cu}, \mathrm{Ni}, \mathrm{Co}, \mathrm{Mn})$ has attracted considerable attention due to their fascinating and diverse crystal structures, which are built out of various extended units of $\mathrm{M}-\mathrm{O}$ and $\mathrm{V}-\mathrm{O}$ polyhedra ${ }^{1-\underline{4}}$ They can have low-dimensional (chain, sheet, honey-comb etc.) to more complex three dimensional structures with intriguing magnetic and electronic properties,$\frac{5,6}{6}$ These pyrovanadates broadly crystallize in two different groups of structures: thortveitite $\left(\mathrm{Sc}_{2} \mathrm{Si}_{2} \mathrm{O}_{7}\right.$ type) or dichromate $\left(\mathrm{K}_{2} \mathrm{Cr}_{2} \mathrm{O}_{7}\right.$ type $) \underline{\underline{4}}$

Among the various members of the group, $\mathrm{Cu}_{2} \mathrm{~V}_{2} \mathrm{O}_{7}$ draws special attention both for its structural and electronic aspects. This compound can crystallize in at least four polymorphic phases (namely $\alpha, \beta, \beta^{\prime}$ and $\gamma$ ) with different lattice symmetry (although all thortveitite type) $\underline{\underline{6}-\underline{\underline{8}}}$ The $\beta$ phase can be described as a spin $1 / 2$ Heisenberg system on a two dimensional (2D) honeycomb lattice $\underline{\underline{6}}$ On the other hand, the $\alpha$ phase is better described by two sets of mutually perpendicular zigzag chains with strong inter-chain magnetic interactions. Here all $\mathrm{Cu}^{2+}$ ions are equivalent with fivefold coordination to oxygen atoms forming a distorted $\left[\mathrm{CuO}_{5}\right]$ polyhedron. Each distorted polyhedron is linked with another two via edge sharing and together they form the two sets of mutually perpendicular zig zag chains (see Fig. 1) in the $b c$ plane $\stackrel{7,9}{\underline{7}}$ Notably, $\alpha-\mathrm{Cu}_{2} \mathrm{~V}_{2} \mathrm{O}_{7}$ crystallizes in the Orthorhombic $F d d 2$ structure and it is the only member of the pyrovanadates to have a non-centrosymmetric crystal structure.

The magnetic and electric properties of $\alpha-\mathrm{Cu}_{2} \mathrm{~V}_{2} \mathrm{O}_{7}$ are equally interesting. The compound undergoes long range magnetic ordering below $T_{C}=35 \mathrm{~K}$ with a spin canted structure $\stackrel{9-11}{-1}$ Recent work by our group identified the compound to be an improper multiferroic with the simul-
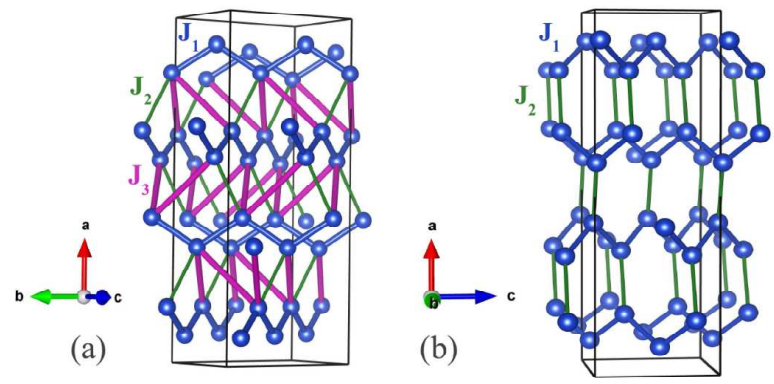

FIG. 1. (color online) (a) Various spin exchange interactions in $\alpha-\mathrm{Cu}_{2} \mathrm{~V}_{2} \mathrm{O}_{7}$ in a conventional unit cell according to modelI. (b) The helical honeycomb network (model-II) of spin exchange comprising $J_{1}$ and $J_{2}$ only.

taneous development of spontaneous electric polarization and magnetization below $T_{C}: 12$ Density functional theory (DFT) based calculations indicate that the magnetism in $\alpha-\mathrm{Cu}_{2} \mathrm{~V}_{2} \mathrm{O}_{7}$ is a consequence of ferro-orbital ordering due to the unique pyramidal $\mathrm{CuO}_{5}$ environment and the origin of the giant ferroelectric polarization is primarily due to the symmetric exchange-striction mechanism. In the DFT calculation, the dominant interaction is obtained between the third nearest neighbors of $\mathrm{Cu}$-ions $\left(J_{3}=-13.61 \mathrm{meV}\right)$ linking the two mutually perpendicular chains. The other sizable interactions are first nearest neighbor $\mathrm{Cu}-\mathrm{Cu}$ linkage $\left(J_{1}=-4.67 \mathrm{meV}\right)$ along the zig-zag chain via oxygen and the second nearest neighbor inter-chain interaction $\left(J_{2}=+4.07 \mathrm{meV}\right)$ along the $a$ axis 


\begin{tabular}{|c||c|c||c|}
\hline \multirow{2}{*}{$J$ 's in meV } & Model-I & \multicolumn{2}{|c|}{ Model-II } \\
\cline { 3 - 4 } & & $J_{1}>J_{2}$ & $J_{2}>J_{1}$ \\
\hline \hline$J_{1}$ & -4.67 & -5.79 & -4.10 \\
$J_{2}$ & 0.8 & -2.61 & -6.31 \\
$J_{3}$ & -9 & - & - \\
\hline
\end{tabular}

TABLE I. Various $\mathrm{Cu}-\mathrm{Cu}$ exchange parameters used for the spin wave simulations. Model-I is based on the exchange interactions obtained by DFT calculations ${ }^{12}$ with required modification, whereas model-II represents the optimized values of $J$ 's as obtained from QMC simulations of the bulk magnetization data. 13

(see Fig. 1 (a)). Evidently, $J_{1}$ and $J_{3}$ are antiferromagnetic (AFM) in nature while $J_{2}$ is ferromagnetic (FM). Model-I, detailed in Table-I, is based on this scenario with required modifications of the exchange parameters to suit our Monte Carlo simulations. On the other hand, a quantum Monte Carlo (QMC) based calculation finds a better fit to the experimental magnetization data considering $J_{1}$ and $J_{2}$ (both AFM in nature) to be the only dominant spin-spin interaction terms, which constitute a helical honeycomb-like 2D spin network (Fig. 1(b)) in the $a c$ plane. $\underline{\underline{13}}$ It is to be noted that in the former scenario (where $J_{3}$ is dominant), the system is magnetically three dimensional, while in the later case (as described by model-II in Table-I) it can be described as a quasi$2 \mathrm{D}$ system with anisotropic magnetic interactions in the honeycomb plane $\left(J_{1} \neq J_{2}\right)$. The spin canting in $\alpha$ $\mathrm{Cu}_{2} \mathrm{~V}_{2} \mathrm{O}_{7}$ is found to be related to the prevailing strong Dzyaloshinski-Moriya (DM) interaction as described by various authors $\stackrel{12-14}{\underline{14}}$

Inelastic neutron scattering (INS) is an important tool to study the low-lying excitations in quantum spin systems, where the dispersion of the excitations and their intensity can provide important information regarding the possible exchange interactions and their relative strengths. In this paper we present INS results from polycrystalline samples of $\alpha-\mathrm{Cu}_{2} \mathrm{~V}_{2} \mathrm{O}_{7}$. The experimental result is corroborated by spin wave simulations based on classical Monte Carlo and linear spin wave theory, which provide support for the dominance of the third nearest neighbor interaction in the system.

The details of the preparation of polycrystalline samples of $\alpha-\mathrm{Cu}_{2} \mathrm{~V}_{2} \mathrm{O}_{7}$ can be found elsewhere $\stackrel{\underline{9}}{\text { The sam- }}$ ple was characterized by powder x-ray diffraction and magnetization measurements. INS measurements were carried out at the MARI time of flight chopper spectrometer $\frac{15}{15}$ at the ISIS pulsed neutron facility, Rutherford Appleton Laboratory, UK. The powder sample of $\alpha-\mathrm{Cu}_{2} \mathrm{~V}_{2} \mathrm{O}_{7}$ was placed in a aluminum foil packet in the form of annulus of diameter $40 \mathrm{~mm}$ and height $40 \mathrm{~mm}$ and sealed in a thin aluminum can, which was cooled in Heexchange gas using a $\mathrm{He}$ closed cycle refrigerator down to a base temperature of $4.5 \mathrm{~K}$. The INS spectra were obtained using different incident energies $\left(E_{i}\right)$ between 8 $\mathrm{meV}$ and $150 \mathrm{meV}$ as well as different temperatures be-

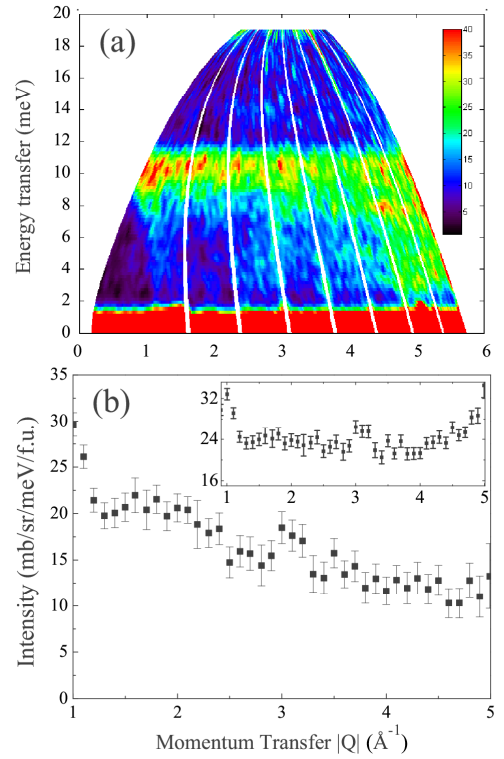

FIG. 2. (color online) (a) 2D map of the scattering intensity of $\alpha-\mathrm{Cu}_{2} \mathrm{~V}_{2} \mathrm{O}_{7}$ as a function of energy transfer $(\hbar \omega)$ and momentum transfer $(|\vec{Q}|)$ obtained at $5 \mathrm{~K}$. The color scale shows the scattering intensity $(S(|\vec{Q}|, \omega)$ in absolute units, $\mathrm{mb} / \mathrm{sr} / \mathrm{meV} /$ f.u. (b) shows the scattering intensity as a function of momentum transfer with phonon scattering corrections applied to the data with the intensity being summed over the energy range $8-12 \mathrm{meV}$ at $5 \mathrm{~K}$. The inset in (b) shows the same data without phonon correction.

tween $5 \mathrm{~K}$ and $100 \mathrm{~K}$. In order to obtain the scattering intensity in the units of cross section, $\mathrm{mb} / \mathrm{sr} / \mathrm{meV} /$ f.u., Vanadium spectra were recorded for the same $E_{i}$ values. The simulation of the spin wave excitations were performed using the SpinW software package $\underline{\underline{16}}$

Fig. 2(a) shows the 2D color plot of the neutron scattering data at $5 \mathrm{~K}$ with an incident energy of $E_{i}=20$ $\mathrm{meV}$. The elastic line contains Bragg peaks, which can all be indexed on the basis of the crystal structure of $\alpha-\mathrm{Cu}_{2} \mathrm{~V}_{2} \mathrm{O}_{7}$. Due to the polycrystalline nature of the samples, the scattering function $S(|\vec{Q}|, \omega)$ is the powder average of the spin-spin correlation function $S(\vec{Q}$, $\omega)$, and it does not carry the information regarding the direction of $\vec{Q}$. A band of scattering intensity is observed around the energy transfer $\hbar \omega=10 \mathrm{meV}$. The magnetic character of the scattering is evident from the decreasing intensity with increasing $|\vec{Q}|$ in the low $|\vec{Q}|$ region $(\leq 3$ $\left.\AA^{-1}\right)$. However, the band of intensity is still present at high $\vec{Q}\left(\geq 3 \AA^{-1}\right)$ values, where one would expect vanishing $S(|\vec{Q}|, \omega)$ had it been purely magnetic in origin. This is quite clearly visible from the inset of Fig. 2(b), where the raw $S(|\vec{Q}|, \omega)$ is depicted as a function of momentum 


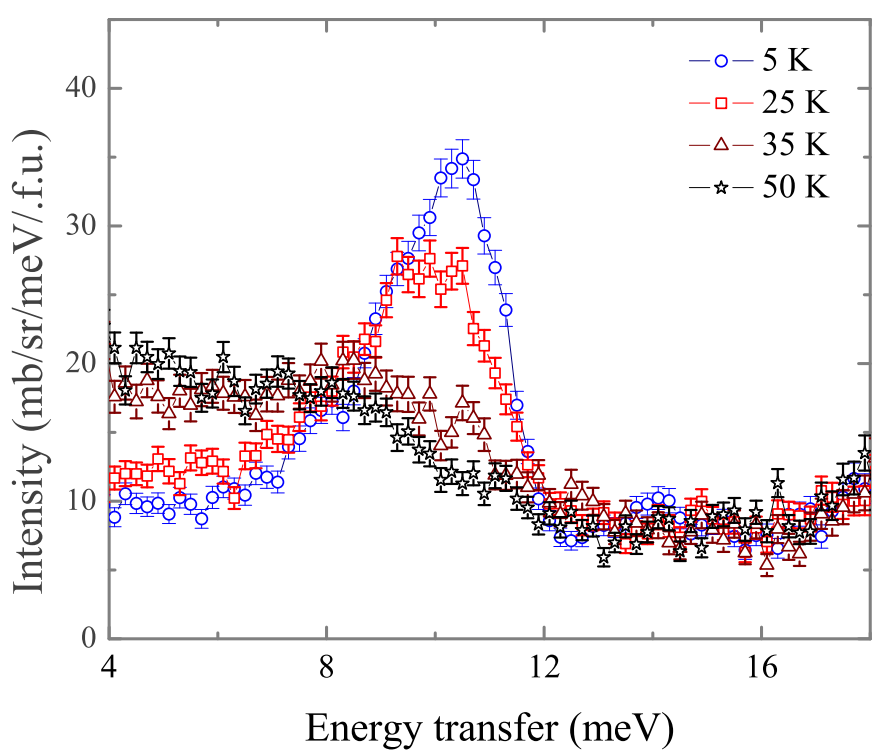

FIG. 3. (color online) (a) Scattering intensity (without phonon correction) as a function of energy transfer for the window of momentum transfer $0-3 \AA^{-1}$ recorded at different temperatures.

transfer for the energy window $8-12 \mathrm{meV}$. We presumed that the high $|\vec{Q}|$ intensity is purely phononic in origin and calculated the vibrational part of scattering at $5 \mathrm{~K}$ using the $300 \mathrm{~K}$ data (well above $T_{C}$ of the sample) after proper normalization with the Bose factor:

$$
\mathcal{B}(\hbar \omega)=\frac{1}{1-\exp \left(-\hbar \omega / k_{B} T\right)}
$$

The vibrational part is subtracted from the measured data and the $|\vec{Q}|$ dependence of the scattering intensity is plotted in the main panel of Fig. 2 (b). The resulting data clearly show diminishing intensity with increasing $|\vec{Q}|$ and establishes the magnetic character of the excitation.

We also investigated the temperature variation of the scattering intensity particularly focusing the low- $|\vec{Q}|$ part $\left(0-3 \AA^{-1}\right)$ as depicted in Fig. 3. The peak around 10 meV diminishes with increasing temperature and almost disappears above about $T_{C}=35 \mathrm{~K}$. This implies that the low- $|\vec{Q}|$ scattering is related to the long range magnetic ordering of the material and that it arises from the spin wave excitations in the ordered state of the material. A small but nonzero scattering intensity is present even in the $50 \mathrm{~K}$ data, which is likely to be due to the short range magnetic correlations in the system. This is in agreement with the previous magnetization studies where the deviation from Curie-Weiss law was observed from below about $80 \mathrm{~K}, \frac{12}{=}$ indicating precursor local spin-spin correlations to the long range magnetic order. It is to be noted that there are also phonon modes near the spin wave energy.

In order to model the experimentally observed mag-
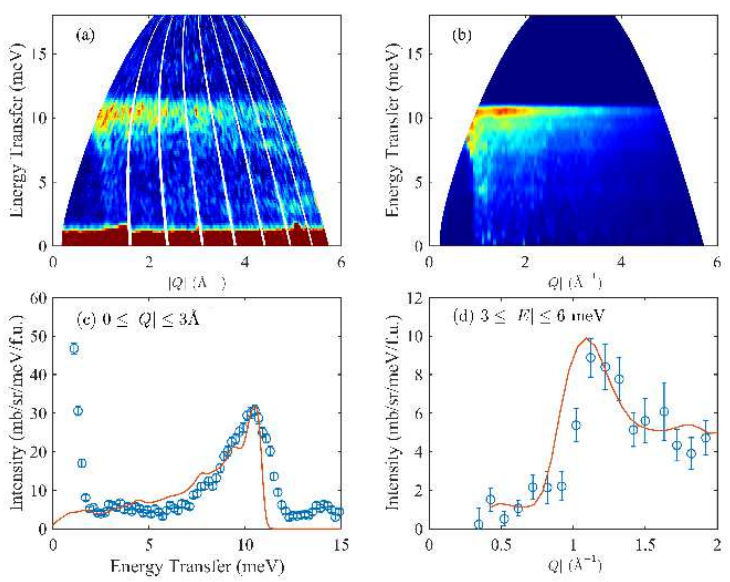

FIG. 4. (color online) (a) and (b) show the 2D color plots of the neutron scattering intensity as obtained from phonon corrected measured experimental data (at $5 \mathrm{~K}$ ) and simulated by the SpinW package respectively. (c) and (d) show the experimental and simulated scattering intensity as a function of energy transfer for the $|\vec{Q}|$ range $0-3 \AA^{-1}$, and as a function of momentum transfer summed over $3-6 \mathrm{meV}$ respectively.

netic scattering, we calculated the spin wave dispersion and corresponding neutron scattering intensity using the SpinW software package. $\alpha-\mathrm{Cu}_{2} \mathrm{~V}_{2} \mathrm{O}_{7}$ contains magnetic $\mathrm{Cu}^{2+}\left(3 d^{9}, S=\frac{1}{2}\right)$ and nonmagnetic $\mathrm{V}^{5+}\left(3 d^{0}, S=0\right)$ metal ions and therefore only the interactions between $\mathrm{Cu}^{2+}$ ions need to be considered. The spin Hamiltonian can be considered as

$$
\mathcal{H}=-\sum_{i j} J_{i j}\left(\overrightarrow{S_{i}} \cdot \overrightarrow{S_{j}}\right)+\sum_{i j} \overrightarrow{D_{i j}} \cdot\left(\overrightarrow{S_{i}} \times \overrightarrow{S_{j}}\right)
$$

where the first part represents the symmetric Heisenberg interaction while the second antisymmetric part is related to the DM interaction. For simplifying the calculation, we have not considered any anisotropy term in the Hamiltonian. For the simulation of the spin wave spectra we used the canted magnetic structure reported by Lee et $a l ., \stackrel{14}{\stackrel{1}{1}}$ and exchange parameters based on model- $1 \frac{12}{12}$ and model-II ${ }^{13}$ as given in table-I. For model-I, as discussed before, the dominant term is $J_{3}$, while in model-II, the exchange paths comprise a helical honeycomb network with $J_{1}$ and $J_{2}$ being the only interaction terms. In the case of model-II, two scenarios may arise, namely $J_{1}>J_{2}$ and $J_{1}<J_{2}$.

At the beginning, we only considered the symmetric exchange terms (namely $J$ 's) and ignored the DM terms ( $D$ 's). In Fig. 4 (a), we have shown the 2D color plot of the experimental INS data where the phonon contribution has been removed. It is clearly seen that the scattering around $10 \mathrm{meV}$ is dominant at low- $|\vec{Q}|$, which is due to its magnetic origin. The simulated scattering data using the $J$ values from model-I (see table-I) is depicted in 

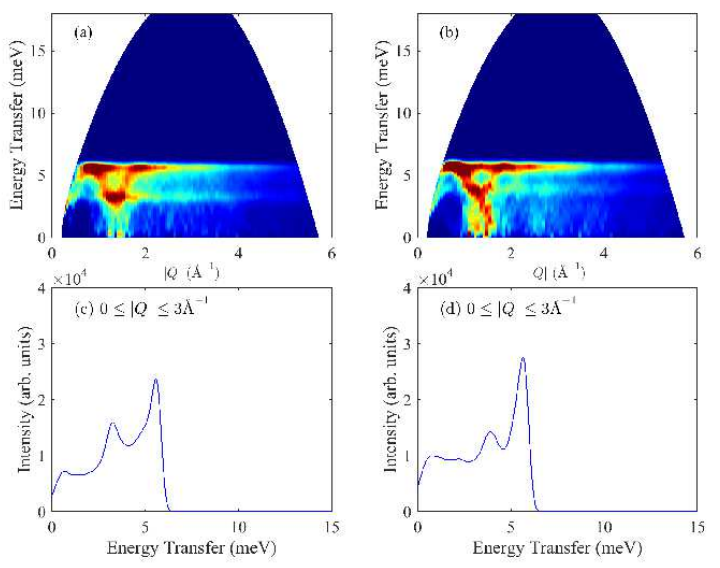

FIG. 5. (color online) Simulated 2D color plot for neutron scattering intensity ((a) and (b)) as well as its energy variation ((c) and (d)) considering the Heisenberg interaction terms $J_{1}$ and $J_{2}$ only (model-II). For (a) and (c) we have considered $J_{1}>J_{2}$ while for (b) and (d) it is $J_{1}<J_{2}$, using the values quoted in table-I.

Fig. 4 (b), and an excellent agreement is obtained when compared with the observed scattering. This is better viewed via the intensity versus energy transfer and intensity versus momentum transfer plots (Figs. 4(c) and 4 (d) respectively). The experimentally determined value of $J_{3}$, to match the zone-boundary energy, is less than that found using DFT $\stackrel{12}{2}$, but the signs of all three interactions are preserved, and $J_{3}$ remains dominant. The $J_{3}$ interaction between two $\mathrm{Cu}$ atoms is through two oxygen $\mathrm{O}$ atoms $(\mathrm{Cu}-\mathrm{O}-\mathrm{O}-\mathrm{Cu})$ belonging to $\mathrm{VO}_{4}$ tetrahedra. Although $\mathrm{V}$ is nonmagnetic, it can expedite the magnetic interaction by electron transfer via its empty $3 d$ level.

The presence of the DM interaction in $\alpha-\mathrm{Cu}_{2} \mathrm{~V}_{2} \mathrm{O}_{7}$ has already been predicted and it is in line with the observed weak ferromagnetism below $T_{C}$. We also performed our simulation considering the DM interaction terms within the framework of model- $\mathrm{I}^{12}$. The introduction of the DM terms does not improve the overall agreement with the experimental data. In order to investigate the strength of the DM interaction and its role in the magnetic scattering, spin wave measurements on a single crystal of $\alpha-\mathrm{Cu}_{2} \mathrm{~V}_{2} \mathrm{O}_{7}$ are highly desirable.

Despite the generally good agreement between the model-I simulation and the experimental data, the linewidth of the cut through the data is found to be wider than the instrumental resolution, which was used for the simulation (Fig. 4(c)). There are several reasons for line-width broadening, such as (i) magnon-electron (ii) magnon-magnon or (iii) magnon-phonon interactions, $\frac{17}{\underline{7}}$ which are not considered in our simulation. $\alpha-\mathrm{Cu}_{2} \mathrm{~V}_{2} \mathrm{O}_{7}$ being a very good insulator, magnon-electron scattering can be neglected. The role of magnon-magnon scatter- ing in line-width broadening can be ruled out as it is only relevant at high temperature close to $T_{C}$. Being a magnetic multiferroic, the likely origin of the line-width broadening is magnon-phonon scattering. In multiferroics where both time and space inversion symmetries are absent, the coupling between low lying magnetic and lattice excitations is relevant $\underline{\underline{18.19}}$ This is also consistent with the exchange-striction mechanism proposed for the system $\stackrel{12}{=}$ The presence of magnon-phonon scattering is also evident from the raw 2D color plot (Fig. 2 (a)), where a high $|\vec{Q}|$ phonon excitation is visible at the same energy as that of the spin wave peak.

We next considered SpinW simulations for model-II, where $J_{1}$ and $J_{2}$ are the only significant exchange terms. The simulated data with two different scenarios $\left(J_{1}>J_{2}\right.$ and $\left.J_{1}<J_{2}\right)$ are depicted in Fig. 5. Clearly the agreement between the experimental and the calculated data is poor here. The magnetic scattering intensity peaks around $5 \mathrm{meV}$ as compared to $10 \mathrm{meV}$ in the observed data.

The present INS study and subsequent simulations on $\alpha-\mathrm{Cu}_{2} \mathrm{~V}_{2} \mathrm{O}_{7}$ indicate that the analysis is compatible with the model-I, having dominant third nearest neighbor exchange interaction $J_{3}$. The presence of strong $J_{3}$, in addition to $J_{1}$ and $J_{2}$, makes the spin model three dimensional, resulting in a long range magnetic ordering below $T_{C}$. The multiferrocity in the $\alpha$ phase is induced by the exchange striction and is therefore directly related to this long range ordering.

In conclusion, we have investigated the pyrovanadate compound $\alpha-\mathrm{Cu}_{2} \mathrm{~V}_{2} \mathrm{O}_{7}$ using inelastic neutron scattering, along with a spin wave analysis. Our INS study reveals well defined dispersive spin wave excitations with a zone boundary energy of $11 \mathrm{meV}$ at $5 \mathrm{~K}$. The spin wave excitations' energy renormalizes with increasing temperature, but the excitations can still be seen at $50 \mathrm{~K}$, which is well above $T_{C}$, suggesting the presence of short range magnetic correlations. Our spin wave analysis has given a reasonably good description of the experimental data. Furthermore our estimated values of the exchange parameters suggest that the third nearest neighbor $\mathrm{Cu}-$ $\mathrm{Cu}$ interaction is the dominant one, in agreement with those calculated theoretically using DFT(see Ref. 12 and references therein). We observe some line-width broadening of the spin wave excitation data which can be attributed to the magnon-phonon scattering, a mechanism that is a prevailing factor for mutliferroicity and magneto-dielectric properties in insulating oxides. The present study can foster research on the magnetic excitations in this class of pyrovanadates, and would generate theoretical interest of the development of a more realistic model to understand the complex magnetic and multiferroic behavior of these materials.

The technical help of Dr. Ross Stewart on MARI spectrometer during the experiment is gratefully acknowledged. A. Bhattacharyya would like to thank DST India for support under Inspire Faculty scheme, while A. Banerjee acknowledges DST-INSPIRE for Ph.D. research 
program. S. Bhowal thanks Council of Scientific and In- dustrial Research (CSIR), India for her research support.
1 M. Touaiher, K. Rissouli, K. Benkhouja, M. Taibi, J. Aride, A. Boukhari, B. Heulin, Mater. Chem. and Phys. 85, 41 (2004).

2 Z. He, J. I. Yamaura, Y. Ueda, W. Cheng, Phys. Rev. B. 79,092404 (2009).

3 Z. He, Y. Ueda, Phys. Rev. B. 77,052402 (2008).

4 Z. He, J. -I. Yamaura, Y. Ueda, W. Cheng, J. Solid State Chem. 182,2526 (2009) .

${ }^{5}$ M. Yashima, R. O. Suzuki, Phys. Rev. B 79,125201 (2009)

6 A. Alexander Tsirlin, O. Janson and H. Rosner, Phys. Rev. B 82, 144416 (2010).

7 C. Calvo and R. Faggiani, Acta Cryst. B31, 603 (1975).

8 S. V. Krivovichev, S. K. Filaov, P. N. Cherapansky, T. Armbruster and O. Y. Pankratova, Can. Mineral. 43, 671 (2005).

9 M. Sánchez-Andújar, S. Yáũez-Vilar, J. Mira, N. Biskup, J. Rivas, S. Castro-García and M. A. Señarís-Rodríguez, J. Appl. Phys. 109, 054106 (2011).

10 L. A. Ponomarenko, A. N. Vasilev, E. V. Antipov and Y. A. Velikodny, Physica B 284-288, 1459 (2000).

11 J. Pommer, V. Kataev, K.-Y. Choi, P. Lemmens, A. Ionescu, Yu. Pashkevich, A. Freimuth, and G. Güntherodt,
Phys. Rev. B 67, 214410 (2003).

12 J. Sannigrahi, S. Bhowal, S. Giri, S. Majumdar, and I. Dasgupta, B 91, 220407(R) (2015).

13 G. Gitgeatpong, Y. Zhao, M. Avdeev, R. O. Piltz, T. J. Sato, and K. Matan,Phys. Rev. B 92, 024423 (2015).

14 Y.-W. Lee, T.-H. Jang, S. E. Dissanayake, Seunghun Lee, and Yoon H. Jeong, EPL 113, 27007(2016).

15 A. D. Taylor, M. Arai, S. M. Bennington, Z. A. Bowden, R. Osborn, K. Andersen, W. G. Stirling, T. Nakane, K. Yamada, D. Welz, in proceedings of: International Collaboration on Advanced Neutron Sources (ICANS XI), Tsukuba, Japan, 22-26 October, 1990.

16 S. Toth and B. Lake, J. Phys.: Condens. Matter 27, 166002 (2015).

17 P. Manuel, D. T. Adroja, Per-Anker Lindgard, A. D. Hillier, P. D. Battle, Won-Joon Son, and M.-H. Whangbo, Phys. Rev. B 84, 174430 (2011).

18 R. V. Aguilar, A. B. Sushkov, C. L. Zhang, Y.J. Choi, S.W. Cheong, and H. D. Drew, Phys. Rev. B 76 060404(R) (2007).

19 A. B. Sushkov, R. V. Aguilar, S. Park, S-W. Cheong, and H. D. Drew, Phys. Rev. Lett. 98, 027202 (2007) 